\title{
Survey of Crowd Detection Algorithms using Wireless Sensor Networks: A Case of People Crowds
}

\author{
Obbo Aggrey \\ Mbarara University of Science and Technology \\ Mbarara, Uganda, East Africa
}

\author{
Nabaasa Evarist \\ Mbarara University of Science and Technology \\ Mbarara, Uganda, East Africa
}

\begin{abstract}
In this paper a survey is carried out on crowd detection algorithms, highlighting challenges and gaps vis-a-avis people crowd detection. The research identifies some of the key capabilities considered invaluable in effective people crowd detection and compares some of the current crowd detection algorithms with regard to these. The results reveal that most algorithms are primarily non-people crowd detection algorithms. While people crowds are intelligent and can easily bypass most of the current crowd detection algorithms. Given the need for detection and management of people crowds, there is great need for specialized algorithms for people crowd detection using effective and non resource intensive methods .
\end{abstract}

\section{General Terms}

Wireless Sensor Networks, Algorithms

\section{Keywords}

Crowd Detection Algorithms, People Crowds Detection

\section{INTRODUCTION}

Wireless Sensor Networks (WSNs) are a category of wireless networks that deploy group(s) of specialized transducers with communication infrastructure intended to monitor, record and communicate results from diverse locations. Dispersed sensor nodes communicate collected data to other nodes or a central node called the sink. Collected data could be records about temperature, humidity, speed/velocity, vibration or chemical concentration. The WSNs can be terrestrial, underground, underwater, multi-media or mobile [3]. The proliferation of Micro-Electro Mechanical Systems (MEMS) has thus led to a varied application of Wireless Sensor Networks (WSNs), from detection of pollution to animal tracking and traffic light control. On the other hand, the dangers arising from unmanaged people crowds can not be over stated.

Sensor based algorithms provide a step by step description of how sensor networks counter some of the problems they try to mitigate while addressing the generic WSN issues of the need for increased battery life spans, robustness and fault tolerance among others. And depending on the objectives of the sensor networks, these algorithms have been deployed as centralized, hierarchical, distributed or hybrid [2] [1]. Fig 1 below is an illustration of major WSN components. In the figure, nodes collect environmental data, then depending on the implementation, route these to the sink via the

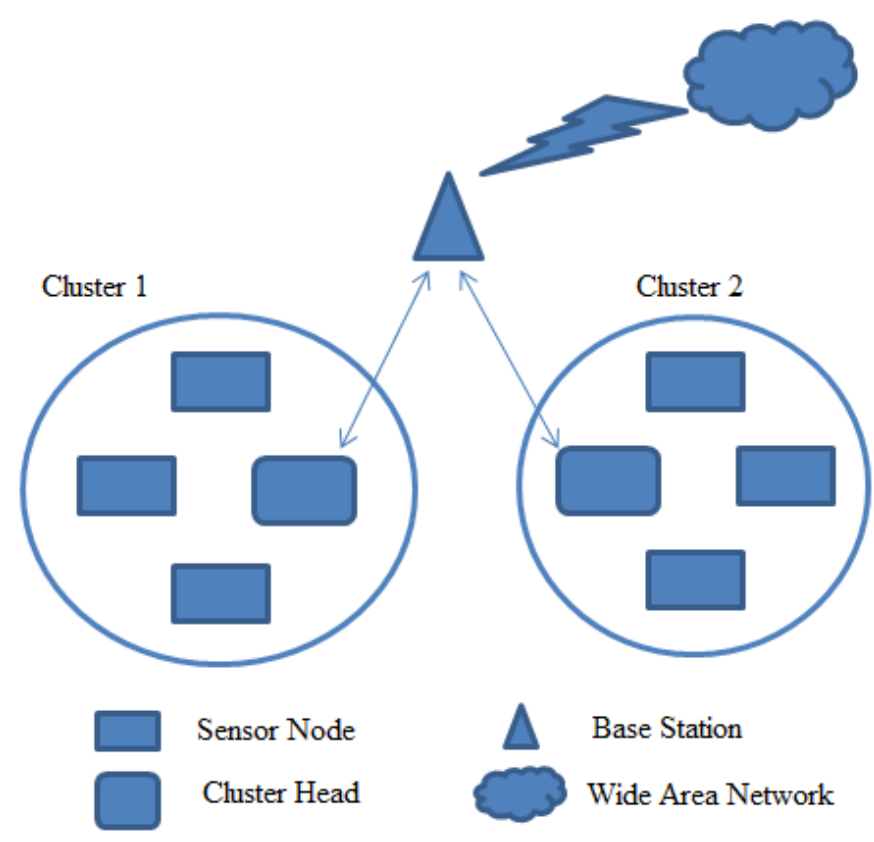

Fig. 1. General Wireless Sensor Network

cluster heads or further through a Wide Area Network (WAN) for remote use or further analysis. The display device that could be connected to any of the networked management devices, provide a visual impression of tracked activities for analysis.

Authors in [8], defined a crowd as a large number of people gathered together in a disorganized or unruly way or a group of people linked by a common interest or activity. It is also considered as a mass or multitude of ordinary people or a large number of things regarded collectively. In this work however, a crowd is defined as the number of targets in a unit contiguous physical space looked at as a single entity. This ensures that a relatively higher number of targets in a very large area is not considered a crowd. Crowd density is thus used to define people crowdedness of an area.

Basing on people crowd behavior during social events, religious gatherings, riots and demonstrations, it is observed that people crowds can result from an increased number of people targets 


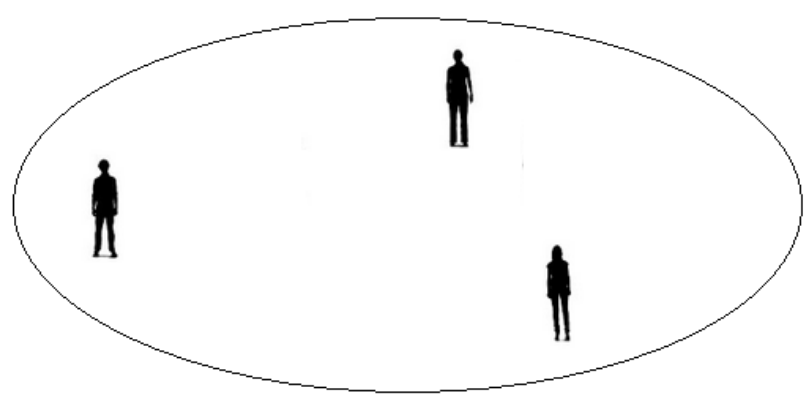

Fig. 2. An example of a sensing region with people targets but with a lower crowd density

within a unit area or groups of targets from different spatial positions converging to an area. There after, the crowds may disperse out in smaller groups. Detection of such characteristics among others can be used to detect people crowd formation or breakout of crowds necessary for people crowd management. In this work, the parameters used are as represented in table 1 , and they include participation, I-O, granularity, $\mathrm{R} /$ Time and $\mathrm{PCD}$ respectively. They are explained below:

(1) Participation: Given the varied areas in application of people crowd detection, people crowd detection algorithms should guarantee participation of subjects.

(2) Indoor and Outdoor support: Research has been done for crowd detection for both indoor and outdoor settings. For a wider application of the people crowd detection algorithms, there should be support for both indoor and outdoor environments.

(3) Granularity: For proper people crowd detection and management, knowledge of target location information is of high relevance.

(4) Real Time: Crowd detection is also of higher value if it has the ability to operate in real time as this also enhances its application.

(5) People Crowd Detection: Finally, in this work, people crowd detection algorithms need to uniquely identify and detect the people crowds.

In WSNs, densely deployed sensor nodes detect crowds by identifying signals unique to the crowd given that presence of people crowds in an area generate distinct characteristic signals. Despite the coverage and power limitations of WSNs, sensor nodes are dispersed over the sensing area. The detection process involves collection, processing, transmission and receipt of sensor data. This ideally comes with communication overheads and thus latency during transmission [19]. In the next section is a survey of some of the crowd detection algorithms.

\section{CROWD DETECTION ALGORITHMS}

This section provides a survey of crowd detection algorithms visavi people crowd.

Crowd detection work has also been carried out in the areas of image processing [12] [10]. A framework for detection of individual songs in bird populations using fixed sensors required a dense uniform distribution of camera enabled sensors nodes in addition to incremental and random configuration that may not be feasible for people detection in urban settings [25]. Image processing are known to rely on crowd features and regression to handle crowds only of a few tens to hundreds of targets. In addition, the complex image processing procedures are resource intensive. While environmental factors like variance in illumination and the extra equipment costs are prohibitive especially for real time image processing [20].

While crowd sourcing approaches rely on participants sharing or uploading crowd related information to a server from were analysis can be made. Different approaches used included the use of Mobile Crowd Sensor (MCS) with a centralized server and the Peer- to Peer data sharing [13] [14]. The MCS approach is limited when it comes to provision of load balancing in the absence of centralized coordination. While the process of uploading data to a centralized server and synchronization are both resource intensive. Crowd sourcing involves sharing of information such as from social media and as such may require provision of incentives to ensure user participation.

Herds and flocks have also been tracked. The Radio Frequency Identification (RFID) based counting systems have been used to track herds of cattle. Authors in [15] studied flock movement patterns, while authors in [4] used accelerometers attached to animals to record pitch angle measurements and velocity estimates in determining herd activity. This however may not be feasible for intelligent people crowds who may have no interest of being detected. It may also not apply to people crowds that are known to abruptly form with limited time to allow for setting up of equipments like accelerometers.

Break through in wireless technologies that has made possible the use of small sized, battery contained and disposable sensors nodes at lower costs, has also propelled the adaptation of Industrial WSNs (IWSNs) in smart parking solutions [16]. Authors in [28] looked at the taxonomy of cloud assisted Internet of Vehicles (IoV) among the vehicle WSN applications. Vehicles however have predefined routes and are not as intelligent as human beings.

The following section is a summary of some crowd detection algorithms using of WSNs.

\subsection{Target Counting through Decentralized Aggregate Management (DAM)}

Research has been done to enable sensors collaboratively determine the number of targets in a region of interest. Authors in [9] get an estimate of the number of herds from the intensity of vibration while the speed at which herds travel is obtained from the frequency of the signal. The targets can either be stationary or mobile. The principle can thus find use in areas that involve study of stationary and mobile targets.

The DAM comprises of a collection of sensors referred to as aggregates that participate in detection of interfering targets in a sensing region. A decision predicate, determines whether a node is to participate in a given aggregate. And for every target, a leader node is elected by sensors exchanging information with their neighbors through one hop broadcasts. Below are the steps followed in DAM determining which nodes participate in the election.

(1) At the beginning of each period, a node makes an announcement indicating its qualification to lead the aggregate. In the announcement, node state parameters are forwarded to neighboring nodes; 
(2) On receipt of the packet, a node compares received values with its own to determine whether to drop or maintain the values basing on which ones score better in the election;

Presence of a crowd is reflected by overlapping peaks while isolated targets are shown by isolated peaks.

Despite its strengths, the target counting approach can not be relied upon for people crowd detection because;

(1) Although this approach has faster convergence and reduced number of communications with neighbors, fault tolerance is still an issue;

(2) The algorithm assumes more detailed sensing capabilities and as a result more resource demanding;

(3) For accuracy to be achieved, sensors need to be even and dense enough which on the other hand yield redundancy and hence wastage of resources;

\subsection{Real-Time Target Tracking Using The Deadline Partition Method.}

The deadline partition method by VigilNet [11], is a real time system intended to track, detect and classify targets in a relatively large-scale sensor network and in a timely manner using the deadline partition method. It was initially designed for spontaneous military activities in remote areas. A characteristic feature in this approach is that the frequency of occurrence of tracked events is low and as a result sensors need not be left awake during times when events are not being monitored. Below is the target detection procedure.

(1) Initial Activation - For purposes of power management, nodes in sleep or non-sentry mode are awakened to form part of the routing infrastructure;

(2) Initial Target Detection - The hardware goes through a response delay before recognizing a target;

(3) Wake-up - The sentry node that detects the target therefore has to "wake-up" the other nodes, creating a wake-up delay;

(4) Group Aggregation - Forms a one-to-one mapping between the nodes and the target, the nodes of which forming a group with a group representative that reaches the Degree of Aggregation (DAG) before forwarding the update to the base;

(5) End-to-End Report - Given the nature of clustered WSNs, several base stations may be used by the leader node to route collected sensor data, hence creating an end-to-end delay;

(6) Base Processing. Ultimately the base station is charged with the responsibility of processing the received reports from the different leader nodes;

The above solution breaks a possible end-to-end time deadline to multiple sub-deadlines incorporated into the detection algorithms, hence deadline partition method. Real time is achieved by ensuring each of the phased sub-deadline time partition are met.

The contributions by these authors is that they address a real time implementable target tracking system. They also investigate tradeoffs between real time performances and parameters such as target speed, detection delay, sentry duty cycle, sensing range, and non sentry duty cycle. In relation to people crowd detection however, this approach is limited because by the following:

(1) Like other real time systems, real time target tracking is resource intensive and may also not be effective given the dy- namics associated with people crowds like crowd formation and movements.

(2) The technique is also associated with a number of delays as highlighted in steps one through six above.

\subsection{Distributed People Counting Algorithm using Pyroelectric Infrared (PIR) Sensors}

The Pyroelectric Infrared Sensors (PIR) emit binary signal that indicate movement, while the signal frequency acts as an indicator of the speed of motion. The signal phase shows the direction of movement. In distributed people counting, distributed sensors record the number of entries and exit to an area to provide an estimate of the number of people in the area.The sensor nodes are connected to a base station through radio waves.

And according to the authors in [24], given space or occupancy $S_{A}$ with predefined entry/exit points $\mathrm{j}$, each observed by nodes $C_{j}$, the people count, $\mathrm{OCC}$ was given by equation 2 below:

$$
O C C_{k}=\sum_{i=0}^{n} \sum_{j}^{N}\left(C_{j, k, \text { in }}-C_{j, k, \text { out }}\right)
$$

And if an estimated output signal was $\mathrm{VCC} / 2$ with no movement, the direction of motion, $\mathrm{S}$ is given by:

$$
S= \begin{cases}\text { left } & \text { if } s \geq(V C C / 2)+v \\ \text { right } & \text { if } s \leq(V C C / 2)-v \\ \text { silent } & \text { Otherwise }\end{cases}
$$

Where $\mathrm{s}$ is the signal amplitude.

The final count algorithm involves the steps below:

(1) Application of the low pass filter to reduce on signal noise;

(2) Identification of signal peaks to determine the key points of the signal;

(3) Identification of patterns in the state of the string using regular expressions and counting the respective traversals while ignoring the peaks in case of a silent state.

The algorithm filters signals to include only low frequency components. While minimum time distance is also defined to eliminate the possibility of having multiple peaks within a short distance and hence a false alarm. And to distinguish between a group of people standing within the sensor line of view and a person actually passing the line, an absolute distance of peaks in terms of signal amplitude is computed.

In [21], the authors used machine learning algorithms to extract people generated information in a room using a single PIR sensor. The generated patterns were used to provide an estimate of room occupancy. It was however found that the single PIR sensor's limited dimensionality restricted its coverage and made it susceptible to occlusion.

Authors in [17] thus proposed two algorithms for estimation of people count; one for considering direction while the other deals with PIR sensor masking. The three step process that constituted the people counting algorithm were segmentation, path selection and people count estimation. To compensate for errors due to masking and insertions, distributed sensor networks were deployed.

Although PIR distributed people counting algorithms require a comparatively lesser number of sensors, the approach together with 
other people counting algorithms like the pedestrian counting systems [5] and estimation of building occupancy [26] are limited in people crowd detection because:

(1) The PIR sensor signals are affected by attenuation as they travel away from the source. This means that they are ineffective for relatively longer distances;

(2) Secondly, implementations of pairs of PIR sensors for recording entry and exit require advance alignment of the sensors and only generate people count which is less than required for effective people crowd detection according to definition of people crowd in section 1 above;

(3) In addition, they have to be placed at predetermined gateways where entry and exit can be closely observed. This may not however be feasible for people crowd detection in outdoor settings where there are unexpected build-up of people crowds that are at times unruly;

\subsection{Device Free Crowd Density Estimation}

Crowd detection requiring volunteers to have devices attached to them can not be relied upon especially when people are not interested in being tracked. Unlike the RFID based systems, in devices free crowd detection, the crowds are not required to have devices attached to them.

Authors in [30] used a devise free system to identify and track entities that do not carry any devise. They looked at techniques for tracking a single intruder. While research by [18] verified the relationship between both the Received Signal Strength Indicator(RSSI) and Link Quality Indicator (LQI) using wireless sensor networks.

In principle, humans are made of $60-70 \%$ water. And whether in motion or stationary, will absorb radio waves. And therefore as the number of people increase between any two nodes, the RSSI average reduces and variance increases. The RSSI is computed from the receiver power that is a factor of transmitter power and the power gains of the receiver and transmitter. The resulting prototype had visual marker detection for place location and web services for collection and distribution of sensor data.

Then authors in [31] build on the concept of the correlation between the radio signal strength and crowd density. The crowd density estimation algorithm used a two step process that composed of the detection and calibration as described below.

(1) The Detection Stage. In this stage, a Received Signal Strength Indicator (RSSI) is analyzed for the WSN environment.

\section{The Detection Algorithm}

A K-means algorithm creates clusters for various crowd densities. And for each of the created cells, a database is built.

While fingerprint algorithm is used to determine whether change as a result of a signal disturbance in the created cell is interesting or not.

A predetermined threshold value, $\Delta=T$ is used to determine whether the status is to be changed from quiet to active. When the status is active, the change is big enough to warrant further analysis. The K-Means algorithm is as follows;

Step 1 - Assignment step: In this step, each observation is assigned to a Voronoi generated cluster with the closest mean.

Step 2 - Update step: Here, the centroid of the observations in each of the clusters is computed.
Step 3 - Computation of initial means: The third step uses foggy partition method to randomly choose $\mathrm{k}$ observations from the data set to compute the initial mean.

(2) The Calibration Stage. The calibration mechanism sets a thresh hold value to reduce noise and deviation in detection. This effort is to reduce errors basing on the spatial temporal correlation principle.

A special distribution is designed to enable receipt of RSSI. The WSN then uses the Collect Tree Protocol (CTP) to ensure all nodes continuously send RSSI information containing packets to the sink.

Calibration Algorithm.

In the calibration process, a threshold is calibrated for each time slot $t_{m}$ and $t_{m-1}$ for the times $t_{0}, t_{1}, \ldots t_{m-1}, t_{m} \ldots t_{n-1}, t_{n}$ together with the respective RSSI values $R S S I_{t_{m}}$ and $R S S I_{t_{m-1}}$. The variance in each of the subareas can then take on the values:

$0: \Delta<10 \mathrm{dBm}$

$1: \Delta>10 \mathrm{dBm}$ and $R S S I_{t_{m}}>R S S I_{t_{m-1}}$

$-1: \Delta>10 \mathrm{dBm}$ and $R S S I_{t_{m}}<R S S I_{t_{m-1}}$

For the sub-areas, sum of RSSI variances is then computed. A positive sum indicates an increase in variance while a negative shows a fall in variance.

Results obtained indicated the RSSI for no person at all, presence of some people, a row or a column of people and a possible crowd. The increasing crowd is reflected by increase in change of RSSI readings.

Conclusion This approach has an advantage of having a better performance compared to camera based approaches that come with high overhead in image processing and suffer from occlusion in crowded scenes. It can also be reused on already deployed WSNs and is low cost [31]. It will however be limited in people crowd detection because of the points below:

(1) The effectiveness of the conventional RSSI under indoor environment approach is negatively affected by reflection, refraction and diffraction caused by both human bodies and other objects.

(2) The approach is not appropriate for people crowd detection as per the definition of people crowds where crowds rapidly build up or break out from already existing people crowds.

(3) The technique was also meant for indoor deployment and as a result may not apply in outdoor environments.

(4) The device free technologies were used for detection of single entities and as such without major modifications will not help in people crowd detection. In addition, they require calibration and incorporation of additional services, hence an increased demand on the already strained WSN resources.

\subsection{Estimating Crowd Densities and Pedestrian Flows Using Wifi and Bluetooth}

Wi-Fi enabled devices periodically broadcast data containing frames even without the intervention of the device user. A count of unique data frames can thus passively be used to estimate the concentration of the devices in a given area. It is assumed that since most people are in possession of these devices, exploitation of this data could be used as a measure of crowd density. 
In bluetooth networking, nodes respond to queries for connection. The targets within the scene of interest respond to these connection requests with responses containing among other things their local name and location. It is on this basis that the devise density and hence crowd density can be estimated.

Authors in [23] estimated crowd densities and pedestrian flows using Wi-Fi and bluetooth. The research provided a tracking system for pedestrian flow estimation. A sixteen days collection of management frames for Wi-Fi and bluetooth was observed through a monitor node placed in a public area and security area to compare the ability of bluetooth and Wi-Fi for pedestrian flow estimation. They looked at pedestrian flow as the amount of people moving one way through an area of interest. This is quite limiting since a crowd may disperse to different directions.

On the other hand authors in [6] went ahead to carry out the estimation basing on Received Signal Strength Indicator (RSSI). A people count was estimated by analysing the effect of a crowd on a direct Line of Sight (LOS) and their scattering effect between two aligned PIR sensors. This was experimented on a small number of people and depended on a pair of PIR sensors fixed well in advance.

(1) The bluetooth technology has coverage as a limiting factor given that bluetooth has a relatively shorter transmission range. This would mean monitoring crowds within close proximity or having multiple devises deployed.

(2) In addition, scanning in bluetooth is resource intensive and given that users devices are multipurpose, users will always disable the bluetooth feature whenever they see it irrelevant.

(3) The fear of receiving unsolicited requests and software bugs means users will only turn on their devices when security is guaranteed.

(4) Technological progress in wireless networks has enabled the Bring Your Own Devise (BYOD) solutions that allows for self configuration and hence use of multiple bluetooth devises by a single user at the same time. Device count may thus not provide a true reflection of the people crowd density.

(5) Use of the system means contending with the longer discovery time for bluetooth as compared with WiFi.

\subsection{Crowd Density Estimation Using Cellular/Mobile Phones}

People crowd detection in Cellular / Mobile phone networks relies on network topology data and Mobile Station (MS) counters in every cell and Location Areas (LAs). The network topology relates to data about cell maps for the different Mobile Network Operators(MNOs)

The technology is based on GSM (General System of Mobile Communications) and UMTS (Universal Mobile Telecommunications System), but can also be adopted by the LTE (Long Term Evolution) technology.

Using a relatively large dataset, spatial and explicit estimation of population densities were produced with more than one billion mobile phones. In addition, they demonstrated how mapping of human population changes over time [7]. Authors in [22] used a hierarchy of intermediate data processing stages employed with each MNO sending their individual Map Counter Datasets (MCD) that included the number of MSs detected together with their corresponding map-counters. Map-counter records for an MNO that constitutes the MCD data sets to a central entity was denoted by $S_{m}$

To estimate the total density $D_{T}$, optionally a union of all the individual MCDs was computed as;

$$
D_{j}=f\left(S_{1}, S_{2}, \ldots\right)=f\left(\bigcup_{m} S_{m}\right)
$$

Where $\mathrm{f}$ is the data processing method.

Because these were so dependent on call logs that can not accurately provide the population estimates compared to mobile data [29], the authors herein had data sets composed of mobile devise ID, Base station location and ID, start and end time of data connection and volume of traffic consumed during a data connection. The authors also provided basic visualization.

Some of the questions answered include: spatial granularity, data collection and fusion and finally how to build a dynamic model to estimate population from cellular data.

In terms of spatial and population coverage, this approach provides diversity across multiple MNOs. It also ensures privacy of critical information since map-counter records are aggregate data and not micro-data. Detection of people crowds may not however be effective because:

(1) MSs today support up to six Subscriber Identity Modules (SIMs). This will therefore be a wrong indicator of people crowd density.

(2) In addition, MSs are either in idle or active state. Most of the time the MS is in idle in a passive state and will not transmit any signals not until there exist intentions to change to another cell. It is therefore difficult to have network detection of these nodes and hence people crowds if it where possible.

(3) Crowd density estimation involves export of records between different MNOs who though may be governed by the nondisclosure laws, on a business perspective this may be critical information to an organization.

(4) The use of mobile data records as recommended by authors in [29], can be a source security concerns since human trajectories are published [27].

\section{CONCLUSION AND RECOMMENDATIONS}

This work, identified five parameters as shown on table 1 below to be considered invaluable in effective people crowd detection and compared how each of the algorithms support the five parameters.In the table, the algorithms either support or do not provide support for the features at least to a reasonable extent. The algorithms represented by DAM, DPM, D/PIR, D-Free W/B, and Phones were Target Counting through Decentralized Aggregate Management, Real Time Target Tracking Using Deadline Partition Method, Distributed People Counting Algorithm using PIR Sensors, Device Free Crowd Density Estimation, Estimating Crowd Densities and Pedestrian Flows Using Wi-Fi and Bluetooth and Crowd Density Estimation Using Cellular/Mobile phones algorithms respectively.

Results show that although work has been done in a number of crowd detection algorithms, this has mainly been in areas of non people crowds. In addition, some of the identified algorithms are limited in people crowd detection either because of their inability 
Table 1. Comparing Crowd Detection Algorithms Vis-a-vis People Crowds

\begin{tabular}{|l|l|l|l|l|l|}
\hline Algorithms & Participation & O-I & Granularity & R/Time & PCD \\
\hline DAM & Yes & No & Yes & No & No \\
\hline DPM & No & Yes & Yes & Yes & No \\
\hline D/PIR & Yes & No & No & Yes & No \\
\hline D-Free & Yes & No & No & Yes & No \\
\hline W/B & No & No & No & Yes & No \\
\hline Phones & No & Yes & Yes & Yes & No \\
\hline
\end{tabular}

to operate outdoor or to guarantee participation, inability to provide location information and importantly the intelligence of people crowds that make it possible for them to sometimes bypass detection. Effort is therefore required for specialized people crowd detection algorithms.

\section{REFERENCES}

[1] Gaddafi Abdul-Salaam, Abdul Hanan Abdullah, Mohammad Hossein Anisi, Abdullah Gani, and Abdulhameed Alelaiwi. A comparative analysis of energy conservation approaches in hybrid wireless sensor networks data collection protocols. Telecommunication Systems, 61(1):159-179, 2016.

[2] Andrea Abrardo, Marco Martalò, and Gianluigi Ferrari. Information fusion for efficient target detection in large-scale surveillance wireless sensor networks. Information Fusion, 38, 2017

[3] Bader A Alyoubi and Ibrahiem MM El Emary. The zigbee wireless sensor netwrok in medical applications: A critical analysis study. Journal of Current Research in Science, 4(1):7, 2016

[4] Claudia Arcidiacono, Simona MC Porto, Massimo Mancino, and Giovanni Cascone. A threshold-based algorithm for the development of inertial sensor-based systems to perform realtime cow step counting in free-stall barns. Biosystems Engineering, 153, 2017.

[5] Matthias Butenuth, Florian Burkert, Florian Schmidt, Stefan Hinz, Dirk Hartmann, Angelika Kneidl, André Borrmann, and Beril Sirmacek. Integrating pedestrian simulation, tracking and event detection for crowd analysis. In Computer Vision Workshops (ICCV Workshops), 2011 IEEE International Conference on, 2011.

[6] Saandeep Depatla, Arjun Muralidharan, and Yasamin Mostofi. Occupancy estimation using only wifi power measurements. IEEE Journal on Selected Areas in Communications, 33(7):1381-1393, 2015.

[7] Pierre Deville, Catherine Linard, Samuel Martin, Marius Gilbert, Forrest R Stevens, Andrea E Gaughan, Vincent D Blondel, and Andrew J Tatem. Dynamic population mapping using mobile phone data. Proceedings of the National Academy of Sciences, 111(45):15888-15893, 2014.

[8] Oxford English Dictionary.

[9] Qing Fang, Feng Zhao, and Leonidas Guibas. Counting targets: Building and managing aggregates in wireless sensor networks. Palo Alto Research Center Technical Report, 2002.

[10] Kang Han, Wanggen Wan, Haiyan Yao, and Li Hou. Image crowd counting using convolutional neural network and markov random field. arXiv preprint arXiv:1706.03686, 2017.
[11] Tian He, Pascal Vicaire, Ting Yan, Liqian Luo, Lin Gu, Gang Zhou, Radu Stoleru, Qing Cao, John A Stankovic, and Tarek Abdelzaher. Achieving real-time target tracking usingwireless sensor networks. In Real-Time and Embedded Technology and Applications Symposium, 2006. Proceedings of the 12th IEEE, volume 10298, pages 37-48. IEEE, 2006.

[12] Yaocong Hu, Huan Chang, Fudong Nian, Yan Wang, and Teng Li. Dense crowd counting from still images with convolutional neural networks. Journal of Visual Communication and Image Representation, 38, 2016.

[13] Changkun Jiang, Lin Gao, Lingjie Duan, and Jianwei Huang. Scalable mobile crowdsensing via peer-to-peer data sharing. arXiv preprint arXiv:1705.05343, 2017.

[14] Robin Kravets, Hilfi Alkaff, Andrew Campbell, Karrie Karahalios, and Klara Nahrstedt. Crowdwatch: enabling innetwork crowd-sourcing.

[15] Patrick Laube, Matt Duckham, and Thomas Wolle. Decentralized movement pattern detection amongst mobile geosensor nodes. Geographic information science, 2008.

[16] Trista Lin, Hervé Rivano, and Frédéric Le Mouël. A survey of smart parking solutions. IEEE Transactions on Intelligent Transportation Systems, 2017.

[17] Kin Sum Liu, Sirajum Munir, Jonathan Francis, Charles Shelton, and Shan Lin. Long term occupancy estimation in a commercial space: an empirical study. In IPSN, 2017.

[18] M Nakatsuka, H Iwatani, and J Katto. A study on passive crowd density estimation using wireless sensors. In The 4th Intl. Conf. on Mobile Computing and Ubiquitous Networking (ICMU 2008), 2008.

[19] Ngoc-Tu Nguyen, Bing-Hong Liu, Van-Trung Pham, and YiSheng Luo. On maximizing the lifetime for data aggregation in wireless sensor networks using virtual data aggregation trees. Computer Networks, 105, 2016.

[20] Eric L Piza, Andrew M Gilchrist, Joel M Caplan, Leslie W Kennedy, and Brian A OHara. The financial implications of merging proactive cctv monitoring and directed police patrol: a cost-benefit analysis. Journal of Experimental Criminology, 12(3):403-429, 2016.

[21] Yordan P Raykov, Emre Ozer, Ganesh Dasika, Alexis Boukouvalas, and Max A Little. Predicting room occupancy with a single passive infrared (pir) sensor through behavior extraction. In Proceedings of the 2016 ACM International Joint Conference on Pervasive and Ubiquitous Computing, 2016.

[22] Fabio Ricciato, Peter Widhalm, Massimo Craglia, and Francesco Pantisano.

[23] Lorenz Schauer, Martin Werner, and Philipp Marcus. Estimating crowd densities and pedestrian flows using wi-fi and bluetooth. In Proceedings of the 11th International Conference on Mobile and Ubiquitous Systems: Computing, Networking and Services, 2014.

[24] Patrick Senti. Distributed People Counting Using a Wireless Sensor Network. PhD thesis.

[25] Erick Stattner, Martine Collard, Philippe Hunel, and Nicolas Vidot. Detecting movement patterns with wireless sensor networks: application to bird behavior. In Proceedings of the 8th International Conference on Advances in Mobile Computing and Multimedia, 2010.

[26] Robert Tomastik, Satish Narayanan, Andrzej Banaszuk, and Sean Meyn. Model-based real-time estimation of building oc- 
cupancy during emergency egress. In Pedestrian and Evacuation Dynamics 2008. 2010.

[27] Zhen Tu, Kai Zhao, Fengli Xu, Yong Li, Li Su, and Depeng Jin. Beyond k-anonymity: protect your trajectory from semantic attack. In Sensing, Communication, and Networking (SECON), 2017 14th Annual IEEE International Conference on, pages 1-9. IEEE, 2017.

[28] Jiafu Wan, Jianqi Liu, Zehui Shao, Athanasios V Vasilakos, Muhammad Imran, and Keliang Zhou. Mobile crowd sensing for traffic prediction in internet of vehicles. Sensors, 16(1):88, 2016.

[29] Fengli Xu, Pengyu Zhang, and Yong Li. Context-aware realtime population estimation for metropolis. In Proceedings of the 2016 ACM International Joint Conference on Pervasive and Ubiquitous Computing, pages 1064-1075. ACM, 2016.

[30] Moustafa Youssef, Matthew Mah, and Ashok Agrawala. Challenges: device-free passive localization for wireless environments. In Proceedings of the 13th annual ACM international conference on Mobile computing and networking, 2007.

[31] Yaoxuan Yuan, Chen Qiu, Wei Xi, and Jizhong Zhao. Crowd density estimation using wireless sensor networks. In Mobile Ad-hoc and Sensor Networks (MSN), 2011 Seventh International Conference on, 2011. 